\title{
Digital base-band rf control system for the superconducting Darmstadt electron linear accelerator
}

\author{
M. Konrad, ${ }^{*}$ U. Bonnes, C. Burandt, R. Eichhorn, P. Nonn, J. Enders, and N. Pietralla \\ Institut für Kernphysik, Technische Universität Darmstadt, D-64289 Darmstadt, Germany
}

(Received 31 January 2012; published 17 May 2012)

\begin{abstract}
The accelerating field in superconducting cavities has to be stabilized in amplitude and phase by a radio-frequency (rf) control system. Because of their high loaded quality factor superconducting cavities are very susceptible for microphonics. To meet the increased requirements with respect to accuracy, availability, and diagnostics, the previous analog rf control system of the superconducting Darmstadt electron linear accelerator S-DALINAC has been replaced by a digital rf control system. The new hardware consists of two components: An rf module that converts the signal from the cavity down to the base-band and a field-programmable gate array board including a soft CPU that carries out the signal processing steps of the control algorithm. Different algorithms are used for normal-conducting and superconducting cavities. To improve the availability of the control system, techniques for automatic firmware and software deployment have been implemented. Extensive diagnostic features provide the operator with additional information. The architecture of the rf control system as well as the functionality of its components will be presented along with measurements that characterize the performance of the system, yielding, e.g., an amplitude stabilization down to $(\Delta A / A)_{\mathrm{rms}}=7 \times 10^{-5}$ and a phase stabilization of $(\Delta \phi)_{\mathrm{rms}}=0.8^{\circ}$ for superconducting cavities.
\end{abstract}

DOI: $10.1103 /$ PhysRevSTAB.15.052802

PACS numbers: 07.05.Dz, 29.20.Ej

\section{INTRODUCTION}

The superconducting Darmstadt linear accelerator (S-DALINAC, [1]) produces electron beams of energies up to $130 \mathrm{MeV}$ in a recirculating scheme. It is operated in continuous wave mode. It uses superconducting niobium cavities at $2 \mathrm{~K}$ with a loaded quality factor of $Q_{L}=$ $3 \times 10^{7}$ for acceleration. Their 20-cell design and the high operating frequency of $3 \mathrm{GHz}$ make them very susceptible for microphonics. In addition, superconducting 2 - and 5-cell capture cavities, one of them supporting acceleration at velocities of $\beta=0.85$, are used inside the injector linac.

Furthermore, room-temperature chopper and buncher cavities are operated to provide the necessary fine structure of the beam. A new polarized-electron injector has been assembled in the accelerator hall [2]. Its bunching system consists of a chopper cavity and a $3 \mathrm{GHz}$ as well as a $6 \mathrm{GHz}$ harmonic buncher. Therefore the rf control system has to deal with different loaded quality factors ranging from some 5000 to $3 \times 10^{7}$ as well as with different operating frequencies.

The target specification for the stability of the accelerating field is a relative error in beam energy of $1 \times 10^{-4} \mathrm{rms}$, mainly for electron and photon scattering experiments

\footnotetext{
*konrad@ikp.tu-darmstadt.de

Published by the American Physical Society under the terms of the Creative Commons Attribution 3.0 License. Further distribution of this work must maintain attribution to the author(s) and the published article's title, journal citation, and DOI.
}

(see, e.g. [3-5] for references). This can be achieved if the error in phase of the oscillating electromagnetic field inside the cavities is kept below $0.7^{\circ} \mathrm{rms}$ and the relative error in amplitude below $8 \times 10^{-5} \mathrm{rms}$ for on-crest acceleration. Since the previous analog rf control system never achieved these values and became more and more unreliable, it has been replaced by the digital rf control system described in this paper.

\section{CONTROLLER-BOARD HARDWARE}

The operating frequencies of 3 and $6 \mathrm{GHz}$ respectively are too high to be controlled directly. The perturbations, on the other hand, are much slower than the rf signal and effectively change only amplitude and phase of the signal. There are two common ways to extract amplitude and phase or $I$ and $Q^{1}$ from the rf signal. The traditional way is to use hardware quadrature (de)modulators that mix the signal down to the base-band directly. This immediately leads to a complex representation of the signal in $I$ and $Q$ coordinates but it has the disadvantage that technical imperfections of the (de)modulator like signal leakage, modulation distortion, etc. have to be dealt with [6].

The intermediate-frequency approach, on the other hand, uses digital quadrature (de)modulation. The rf signal is mixed down to an intermediate frequency in the low $\mathrm{MHz}$ range that is sampled directly by an analog-to-digital

\footnotetext{
${ }^{1} I$ and $Q$ are the coordinates of a Cartesian coordinate system rotating in the complex plane with the frequency of the master oscillator.
} 
converter (ADC). The quadrature (de)modulation is done digitally. The down side of this approach is that the occurrence of image frequencies complicates separation of different eigenmodes of the cavity (especially if they have little spacing). In addition to that the digital clock needs to be synchronized to the rf master oscillator with very low jitter. Last but not least, the much faster ADC has a lower precision than ADCs used for base-band applications. There are ways to address these problems but things get a lot more complicated with higher operating frequencies and higher accuracy. That is why the base-band approach has been chosen for the S-DALINAC's rf control system.

The hardware of our rf control system is split into two parts: A frequency-dependent rf board containing the quadrature (de)modulator and a frequency-independent field-programmable gate array (FPGA) board processing the signals (see Fig. 1). All hardware components have been developed in-house.

In addition to the quadrature (de)modulator, a separate power detector is located on the rf board which improves the accuracy of the amplitude measurement. The output signal of this detector is proportional to the envelope of the input signal and thereby proportional to the field in the cavity. The chosen amplitude detector has a much better linearity than the $I / Q$ demodulator. See [7] for a detailed description of the rf front end.

The analog signals are low-pass filtered on the FPGA board before they are digitized to avoid aliasing effects. The filters used for this purpose are third-order $\pi$ filters with an edge frequency of $\approx 100 \mathrm{kHz}$. This filtering step is very important because the $19 / 20 \pi$ mode of the 20 -cell cavities is only separated by $700 \mathrm{kHz}$ from the $\pi$ mode used for acceleration. The chosen filter design ensures that the adjacent mode is suppressed by $55 \mathrm{~dB}$ while the phase shift introduced by the filter is negligible for microphonic frequencies (up to $10 \mathrm{kHz}$ ).

To meet the specification with respect to accuracy high-linearity 18-bit ADCs (Analog Devices AD7982) are used. They are operated at the maximum sampling rate of $1 \mathrm{MS} / \mathrm{s}$ to keep latency low and to allow the system to detect the signal of a detuned cavity operated in selfexcited-loop mode.

The FPGA used on the controller boards is a Xilinx Spartan 6 (XC6SLX45). Roughly 25\% of its resources are used up to now leaving enough space for future extensions of the algorithm.

The digital $I / Q$ output signals are converted back to analog using digital-to-analog converters (DACs) (Analog Devices AD9747). Low-pass filters are used to construct smooth analog signals that are modulated on the rf signal and sent to the rf amplifier.

\section{CALIBRATION}

\section{A. Demodulator}

The output $I$ and $Q$ signals of the demodulator suffer from DC offsets that may result in wrong values for the input phase. To minimize this error, the demodulator has to be calibrated. As constant offsets have the biggest impact on small input signals, the calibration is done without an input signal (50 $\Omega$ load connected to the rf input). Since the DC offsets are small (less than a few percent), they can be eliminated digitally inside the FPGA.

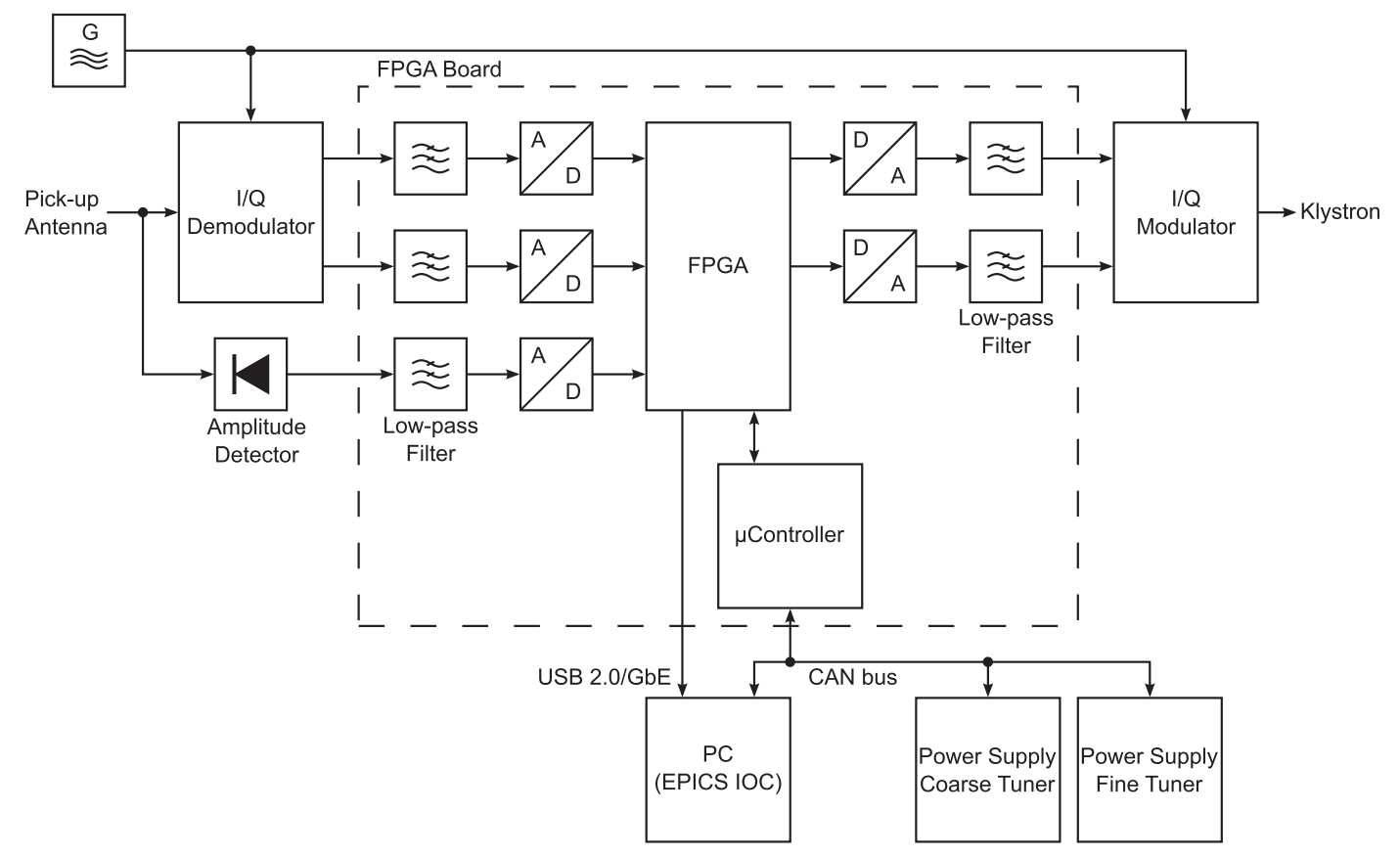

FIG. 1. Overview of the hardware components of one channel of the rf control system. 
In addition to DC offsets, the gains of the $I$ and $Q$ paths of the demodulator might differ slightly. An appropriate way to detect this is by applying an $\mathrm{rf}$ signal to the input of the rf board that differs by approximately $1 \mathrm{kHz}$ from the local oscillator. This results in an ellipse in the $I / Q$ plane that can be visualized with a software oscilloscope. Its eccentricity is compensated for by adjusting the gain factor of the signals inside the control algorithm.

Each combination of rf module and FPGA module has to be calibrated individually after fabrication. So far, recalibration has not been necessary.

\section{B. Modulator}

A DC offset on the $I$ and $Q$ inputs of a vector modulator leads to insufficient carrier suppression. If the carrier signal is strong enough, this can make self-excited loop operation impossible because the loop locks to the phase of the carrier rather than to oscillate freely (self-locking). This is avoided by modulator calibration.

Comparison of the magnitude of the carrier and the signal is achieved by a single sideband sine modulation. To this end, a rotating vector in the $I / Q$ plane is modulated with $f \approx 1 \mathrm{kHz}$ on the carrier while observing the $\mathrm{rf}$ output with a spectrum analyzer. The rotating vector is generated in the FPGA using a counter providing a sawtooth signal as phase input for a CORDIC block (described below). The calibration is done by fine-tuning the $I$ and $Q$ offsets for optimal carrier suppression.

In a second step, an elliptical deformation caused by an asymmetry of the modulator is compensated. This is done by adjusting the gains for $I$ and $Q$ until the magnitude of the image frequency is minimized. All these calibration steps are done completely digitally, and the results can be saved by the accelerator's control system.

\section{CONTROL ALGORITHMS}

The different loaded quality factors of our cavities make different control algorithms necessary. The low- $Q_{L}$ roomtemperature copper resonators have a broad resonance and are relatively rigid: Only slow drifts of the field caused by fluctuations of the ambient air and cooling water temperature are important. Therefore a relatively simple generator-driven resonator control algorithm is sufficient.

In contrast, the superconducting cavities have a high loaded quality factor. Because of their 20-cell design they have low flexural stiffness and thus are very susceptible to microphonics. Hence, the controller has to operate much faster. In addition, it is important that the cavity can be excited even if it is detuned. This is accomplished with the self-excited loop algorithm.

\section{A. Generator-driven resonator}

Figure 2 shows a simplified flow chart of the generatordriven resonator (GDR) algorithm as it is used for our copper cavities. The $I$ and $Q$ input signals are transformed into polar coordinates and back to Cartesian coordinates in the FPGA by the CORDIC algorithm [8].

After conversion to polar coordinates phase control is achieved by an integral controller only. The integrator is necessary to eliminate steady-state offsets. Since there are no fast perturbations, but only slow drifts, no proportional controller is needed for these cavities.

The amplitude controller follows a similar design, but only positive amplitude values must be fed into the output CORDIC, as negative values are unphysical and would cause ambiguities after the CORDIC transformation.

A multiplexer allows to switch between the following modes of operation: (i) Constant output amplitude: In this mode only the phase controller is active while amplitude feedback is turned off. Instead the output magnitude can be adjusted directly by the operator. (ii) Amplitude control switched on: Phase and amplitude feedback are both activated. This mode is used for accelerator operation.

Unlike the self-excited loop algorithm there is no need for complicated commissioning: A cavity on standby can be switched on straight into mode (ii) without any further user interaction.

\section{B. Self-excited loop}

In contrast to the GDR algorithm a self-excited loop (SEL, $[9,10]$ ) oscillates freely at a frequency that is determined by the eigenfrequency of the resonator and the loop

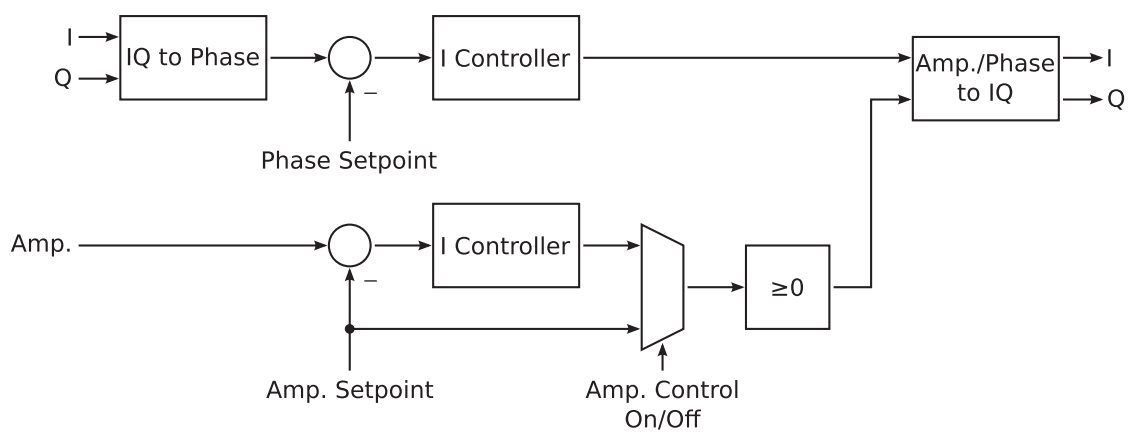

FIG. 2. Simplified flow chart of the generator-driven resonator control algorithm used with the room-temperature cavities. Amplitude and phase of the output signal are generated by the integral controllers. 
phase. Thus, this frequency can be locked to the frequency of the master oscillator by a controller that tunes the cavity's resonant frequency and/or applies an additional phase shift (phase-locked loop). The eigenfrequency of the superconducting cavities can be shifted by means of a tuning system: A magnetostrictive actuator allows continuous fine-tuning whereas a motor tuner provides a larger tuning range.

The advantage of the SEL is that it immediately excites the cavity even if the cavity's eigenfrequency is detuned by many bandwidths. Furthermore, the controller can recover from a breakdown even in the presence of (in case of the S-DALINAC static) Lorentz force detuning that might prevent a GDR from restarting oscillation.

Figure 3 shows a block diagram of the chosen implementation of the SEL algorithm. Again the $I$ and $Q$ signals are transformed into polar coordinates and back to Cartesian coordinates by the CORDIC algorithm. Polar coordinates allow distinct controllers and parameters for amplitude and phase.

To avoid excitation of mechanical eigenmodes, the eigenfrequency of the resonator can only be tuned slowly. An integral controller is used for eigenfrequency control because it eliminates steady-state frequency offsets by tuning the SEL to exactly match the frequency of the master oscillator. Fast perturbations, on the other hand, are compensated for electrically. Instead of an actuator that shifts the phase, a microphonics compensator [9] is used. This block adds an orthogonal correction vector to the input vector. If the length of this correction vector is proportional to the phase error, the microphonics compensator has the inverse transfer function of the resonator. Thus, phase and amplitude errors, which always occur correlated if they are caused by detuning of the cavity, are corrected in a single step. The microphonics compensator is driven by a proportional controller.

In addition to the microphonics compensator, an amplitude controller is needed to compensate for perturbations of the field amplitude. Again this is done by a proportional controller for fast perturbations that cannot be removed completely by the microphonics compensator, whereas an integral controller removes the steady-state offset. The amplitude controller also compensates for changes of the accelerating field strength caused by beam-current fluctuations.

The motor tuner is equipped with a three-step switching controller that automatically activates the motor if the finetuner approaches its limits. The motor tuner causes vibrations and increases the amount of microphonics the $\mathrm{rf}$ control system has to compensate. Therefore a hysteresis ensures that the cavity is always tuned to the middle of the fine-tuner's controlling range once the motor has been activated. Because of this behavior the motor is rarely activated. In fact, it usually does not happen during beam time. The motor tuner is inhibited if the rf signal is switched off by the operator or the rf interlock to avoid that small offsets or noise detune the cavity.

The following modes of operation are possible: (i) Constant output magnitude: The rf control system acts as a limiter. The phase is passed through without any feedback. This mode can be used to test if the SEL oscillates freely (no self-locking). (ii) Microphonics compensation: The microphonics compensator is switched on while the output amplitude is otherwise kept constant. This mode is only useful in situations when the cavity is tuned to the frequency of the master oscillator. (iii) Microphonics compensation and resonance control: Like mode (ii), but the cavity is tuned to the reference frequency automatically.

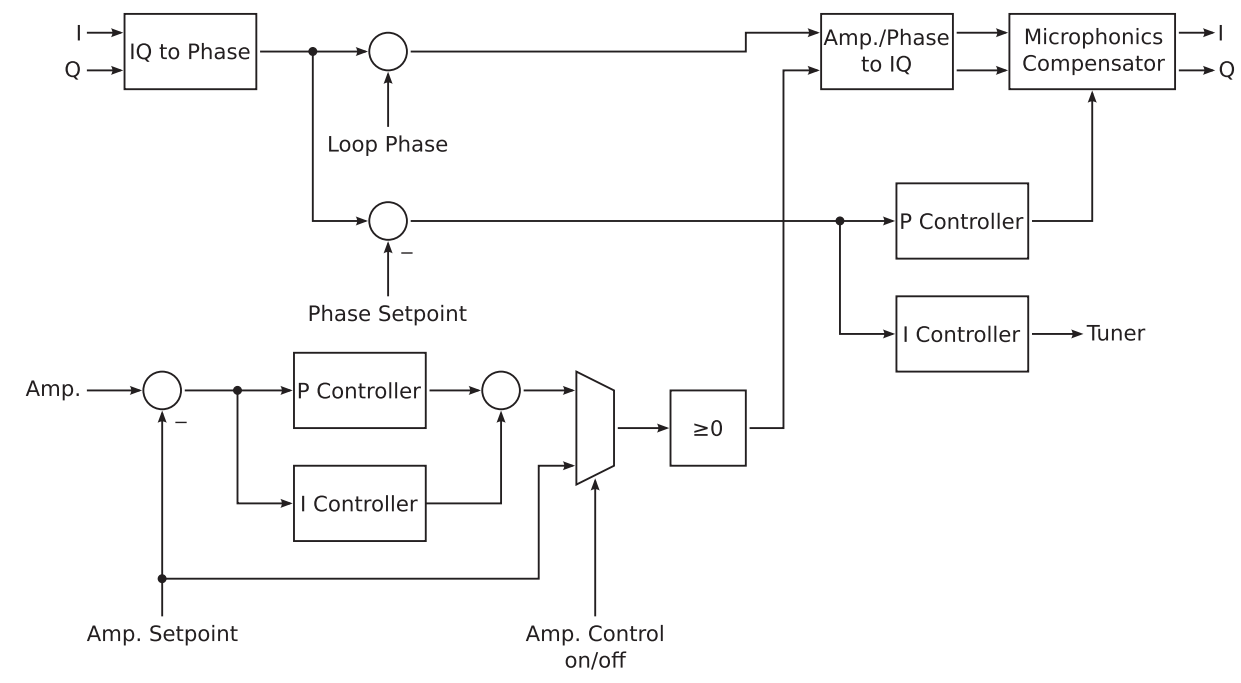

FIG. 3. Simplified flow chart of the self-excited loop control algorithm used with the superconducting cavities. In contrast to the GDR algorithm, the SEL algorithm passes through the phase-shifted input signal. A correction to this signal is applied by the microphonics compensator. 
(iv) Microphonics compensation, resonance control, and integral amplitude feedback: Like mode (iii), but the integral amplitude controller regulates the electric field strength to meet the set-point on average. (v) Full phase and amplitude feedback: In addition to mode (iv) the proportional amplitude controller is activated. This mode is used for accelerator operation.

When operated for the first time, the cavity needs to be tuned to resonance manually. This is done by using a network analyzer synchronized to the master oscillator. Alternatively, the cavity's eigenfrequency can be tuned slowly in mode (i) while keeping the transmitted power at its maximum by adjusting the loop phase. In this case, no further equipment is needed. The progress of the tuning process can be monitored by the frequency of the $I$ or $Q$ signal. After tuning the cavity to the reference frequency, the loop phase is adjusted for maximum transmitted power. This corresponds to a total phase shift along the open loop that is a multiple of $2 \pi$. As long as the operating frequency of the cavities remains the same, there is no need to retune the loop phase later on.

The next step is to activate the microphonics compensation [mode (ii)]. This compensates for a possible small deviation of the resonator from the reference frequency by increasing the output power as well as shifting the phase of the output signal to stabilize the phase of the field. After switching to mode (iii), the integral phase controller ensures the cavity's eigenfrequency matches the reference frequency. This removes the steady-state offset on the input of the microphonics compensator and thereby reduces the amount of rf power that is needed to drive the cavity.

Finally, amplitude feedback is activated [modes (iv) and (v)]. The proportional controller stabilizes the field on a fast time scale whereas the integral controller removes the steady-state offset.

The described procedure has to be followed only at firsttime operation. After a normal rf shutdown (leaving the loop phase and tuning unchanged) the controllers recover automatically.

\section{FPGA AND SOFT CPU}

The control algorithms mainly use simple arithmetic operations like multiplications and adding operations. In addition to that, CORDIC blocks are used for the transformation from Cartesian coordinates $(I$ and $Q)$ to polar coordinates (amplitude and phase) and back.

The control algorithm is implemented as a program for a self-developed soft CPU that is placed in the FPGA. The CPU is highly optimized for the implementation of $\mathrm{rf}$ control algorithms. Its arithmetic logic unit uses 18-bit operands in order to fully exploit the accuracy of the ADCs. The instruction set is reduced to the operations needed for rf control algorithms. The CPU does not use complex memory access but only variable registers and parameter registers. Parameter registers are used for control parameters that are adjusted by the operator. They are read-only for the soft CPU but read/write for the microcontroller which connects the controller board to the PC. In contrast, variable registers used for intermediate results are read-only for the microcontroller but read/write for the soft CPU. This concept together with a two-staged pipeline allows a high clock rate of $80 \mathrm{MHz}$. Complex operations like the conversion from Cartesian coordinates into polar coordinates and back are based on Xilinx CORDIC IP cores [11] that can be used from the CPU and that are pipelined as well (about 30 stages).

The CPU provides several 36-bit accumulating registers that can be used for implementing integral controllers. Only the 18 most significant bits are used for further processing, but all 36 bits are considered for the accumulated sum. This allows integral controllers with time constants of up to $\approx 1 \mathrm{~s}$ to be realized.

To avoid discontinuities, all operations are available in a normal and a clipping variant. The latter clips the signal to the maximum/minimum 18-bit value if the result of an operation exceeds the range. The normal variant of the instructions is typically used for phase operations with cyclic boundary conditions whereas the clipping variant is used for calculations with amplitude or $I / Q$ values which should have a saturating behavior.

Using a soft CPU for the control algorithm has advantages in diagnostics because all variables (and thereby all intermediary results) are stored in fixed registers (block random-access memory) where they can be read out easily to allow for diagnosis on bit level. In particular, there is no need for 18-bit data paths and huge multiplexers throughout the whole FPGA that would consume a great deal of the resources. In addition, the soft-CPU approach speeds up the development of the control algorithms. No timeconsuming synthesis of the VERILOG code is necessary after the control algorithm has been changed. On the other hand, the serial processing of the data reduces the performance. For most practical purposes this penalty is small since the latency between ADC and DAC is determined mainly by the CORDIC blocks. The latency caused by the complete control algorithm is approximately $1 \mu \mathrm{s}$.

\section{SLOW CONTROL}

Apart from the control algorithm itself there are no functions that need to be carried out in real time. This especially includes monitoring and adjusting parameters of the hardware and the control algorithm by the operator. Hence, all controller boards are connected to a standard PC server via CAN bus. The microcontroller connecting the FPGA boards to the CAN bus runs NUT/OS, an open-source real-time operating system providing cooperative multithreading [12]. In addition to communicating with the $\mathrm{PC}$, it sends commands directly to the fine and coarse tuner power supplies via CAN 
bus. A three-step controller implemented in the microcontroller activates the motor tuner once the fine-tuner approaches its limits.

The PC runs Linux and an EPICS input output controller (IOC) that is connected to the CAN bus via a device support that uses the SOCKETCAN network stack [13] included in recent Linux kernels (since 2.6.25). SOCKETCAN provides access to the CAN bus via network devices (BSD sockets) which can be accessed by multiple applications at the same time (e.g. the IOC and a CAN sniffer). Furthermore, it acts as an abstraction layer making the device support independent of a specific CAN card or hardware vendor: All CAN cards having a SOCKETCAN driver are supported. In total, the IOC provides several thousand records for all $16 \mathrm{rf}$ channels, largely related to diagnostics.

The operator interface has been implemented with the BOY plug-in [14] included in CONTROL SYSTEM STUDIO [15]. Figure 4 shows a screenshot of a display providing an overview of all control loops and their most important controls. Further controls are available on control algorithm specific displays that contain all parameters for the selected controller.

\section{DIAGNOSTICS}

In addition to the ability to change control parameters, the operator needs detailed information on how the control loops behave. This requires much higher data rates than the
CAN bus can handle. Therefore the FPGA boards provide fast diagnostic data via USB 2.0. Eight signals with a resolution of 16 bits are continuously transferred to the PC with the full sampling rate of $1 \mathrm{MS} / \mathrm{s}$. The two least significant bits of the full 18 bit result are available as separate channels.

During operation eight controller boards are installed in a crate together with a concentrator card. The concentrator card uses an FPGA to collect the data from the controller boards and streams the selected signals to the PC via its own USB 2.0 interface (cf. Fig. 5). Two crates can be coupled through a parallel low-voltage differential signaling interface. In this configuration, one concentrator module acts as master and the other as slave. Signals from different controller boards and crates can be transferred at the same time.

In addition to the eight channels with full sampling rate, the master concentrator card provides a stream of all 1024 signals (16 controller boards with 64 signals each) on a second USB 2.0 interface. This data is transferred with a reduced sampling rate of $2 \mathrm{kHz}$ and is intended for monitoring. Both USB data streams end at the PC where the IOC reads the data via a specially developed device support.

The data streams are forwarded by the diagnostic server process to the connected clients via TCP connections. Typical client applications include a software oscilloscope [16] that has been extended with a data source plug-in that reads data over the network from the IOC as well as a

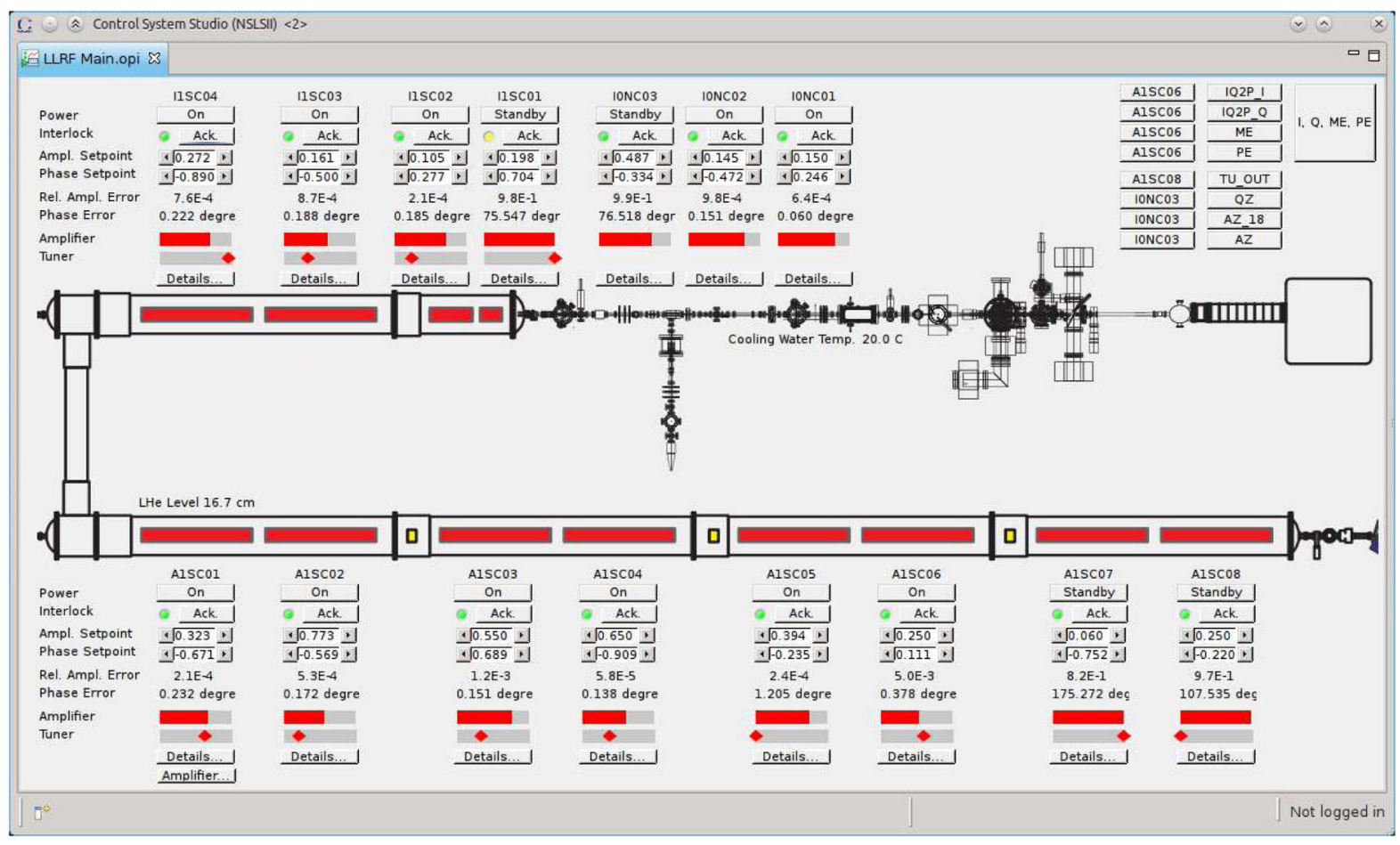

FIG. 4. Screenshot of the main display of the rf control system's operator interface. It only provides the most frequently used controls and links to further displays for details. 


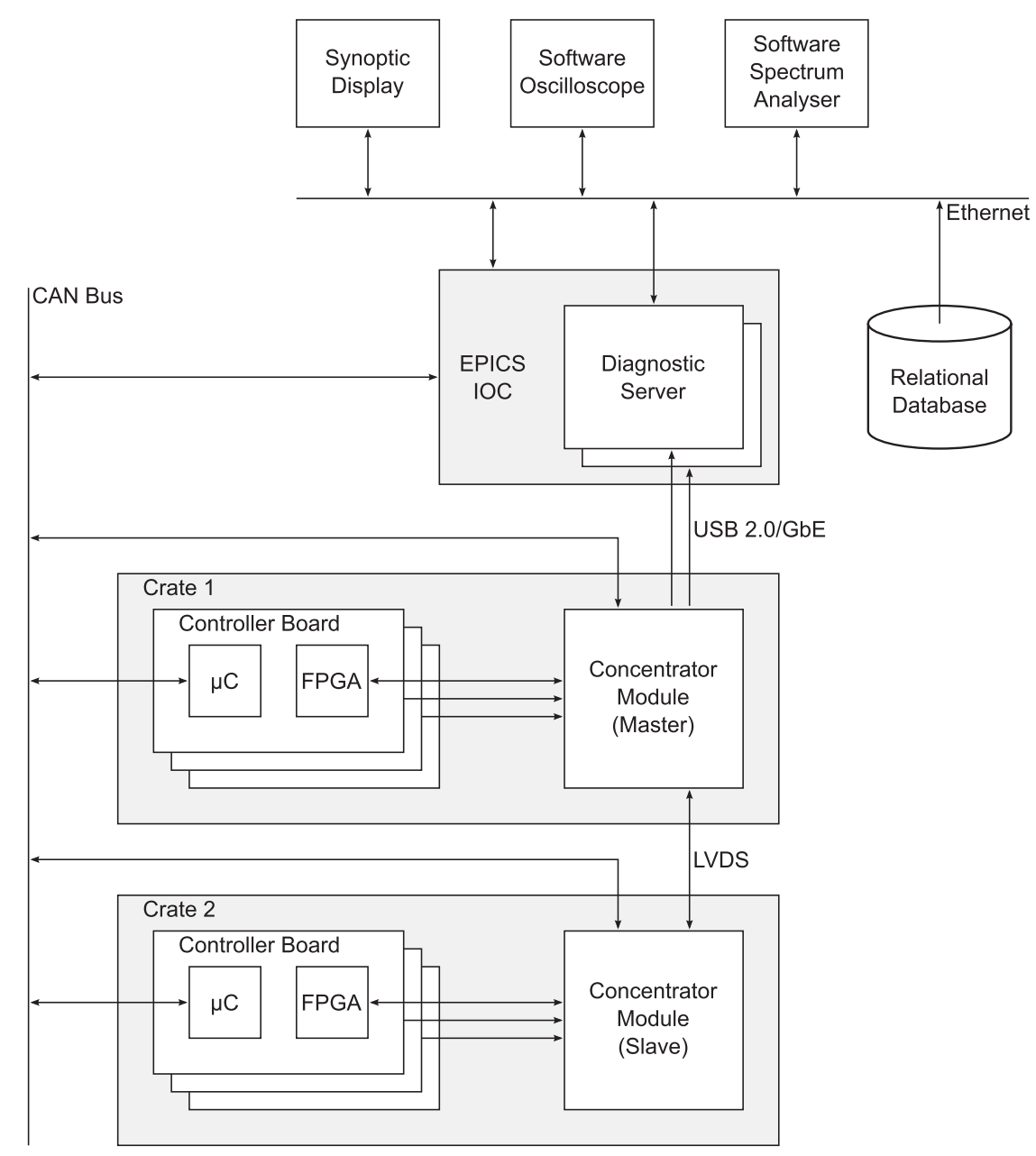

FIG. 5. Data flow between controller boards, concentrator modules, diagnostic server, and clients.

software spectrum analyzer [17]. Data recording is possible with standard Unix tools (e.g., NETCAT).

In addition to forwarding the stream of data, the slow data stream is fed into EPICS records by the device support. It provides mean values of all signals every $0.1 \mathrm{~s}$ which are used for monitoring.

Those values may detect if a controller is out of lock, but they do not provide precise information about the performance of the cavity and its controller. In order to quantify the variations of the field in the cavity, the rms errors of the amplitude and phase error signals are calculated. Instead of determining the errors from the fast streaming data at the PC, thereby occupying all eight diagnostic channels, the rms calculation has been moved partly into the FPGAs of the controller boards. They have the whole data stream available and as a side effect relieve the PC from processing work by carrying out the most time-consuming part of the calculation. The FPGAs calculate the squares of the signals for each ADC sample and sum $2^{17}$ of them up which corresponds to a time of roughly $0.1 \mathrm{~s}$. The result is transferred to the PC via
CAN bus. The EPICS IOC calculates the square root of the sums and scales the values to finally get an absolute error in degrees for the phase or a relative error in case of the amplitude, respectively.

\section{SOFTWARE AND FIRMWARE DEPLOYMENT}

The rf control system has been implemented as soon as the major components allowed stable operation. Within the concept of continuous integration, new features and corrections have been integrated through frequent recompilation and deployment of the software to the PC server and of the firmware to the microcontrollers and FPGAs. This needs to be done in a repeatable fashion and has been achieved by using the DEBIAN FULLY AUTOMATIC INSTALLATION (FAI, [18]) to setup the PC server over the network. A virtual machine is used for testing whether all packages are installed successfully before the production machine is reinstalled. Because most software components are automatically built by a build server and distributed as binary Debian packages, changes can be deployed to the 

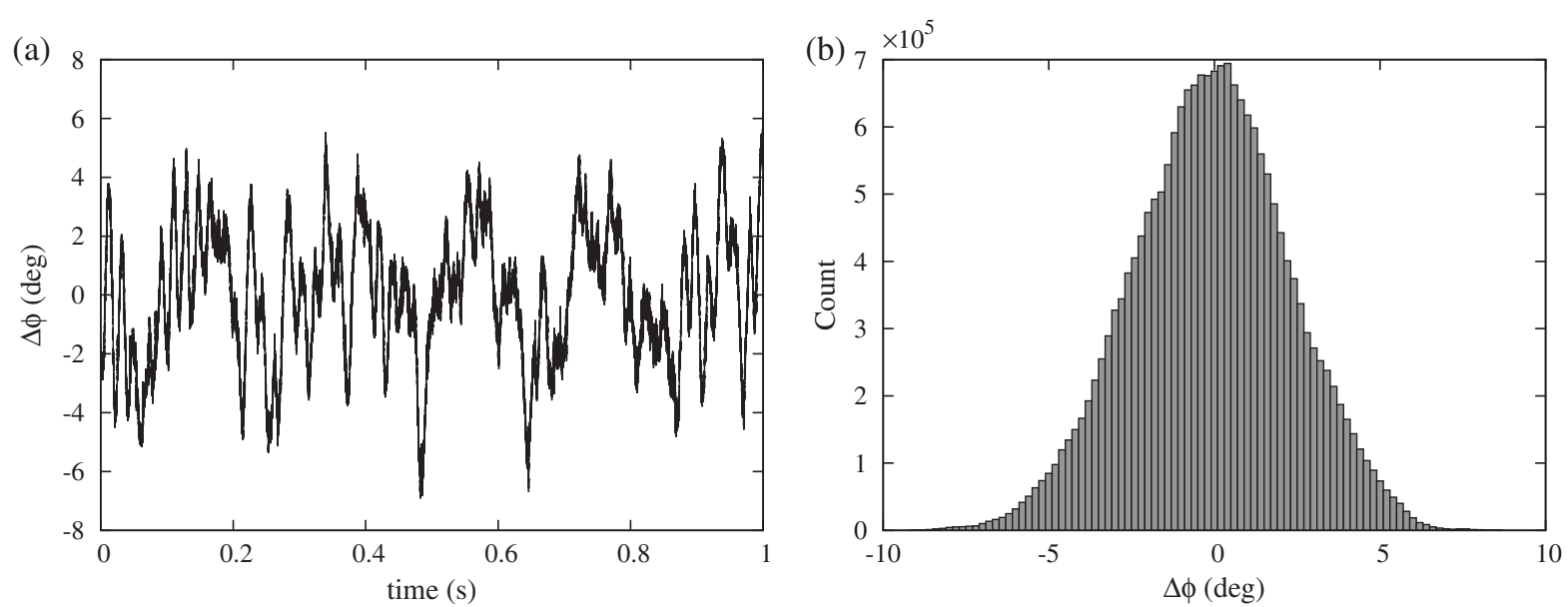

FIG. 6. Phase error of a freely oscillating superconducting cavity in SEL mode. Sinusoidal structures of several frequencies are superposed resulting in a nearly Gaussian distribution of the phase error.

test system or to the production system within minutes. A complete installation of the server takes about 10 minutes. Hence, downtimes of the accelerator of as short as half an hour can be used to upgrade the entire rf control software. Updates of the IOC are possible even during operation because the controller boards run independently from the $\mathrm{PC}$.

The IOC configuration is generated automatically from a relational database and a set of templates during installation. This way a quick recovery from a PC failure is warranted. One spare machine is sufficient for all control system PCs running Linux.

First-time programming of a virgin FPGA board or tuner power supply has to be carried out via the JTAG chain. This interface allows in-circuit programming and in-circuit testing of all reprogrammable components. Later on, microcontroller firmware images of the FPGA boards and the tuner power supplies can also be updated via CAN bus in the field. A boot loader developed in-house makes it possible to recover from broken firmware images without attaching a programmer. The configuration of the FPGAs can be written via CAN bus as well as via JTAG. A multiboot functionality provides a fallback to a second "golden" image for the FPGA in case of a broken primary bitstream [19]. These measures together with revision control of all components make a significant contribution to reduce the downtime of the rf control system.

\section{MEASUREMENTS}

To make sure that the rf control system meets the specifications, its performance was studied in several measurements. Thereby it is essential to take the full frequency range of perturbations into account to get the total error. Pressure fluctuations of the helium bath in the sub- $\mathrm{Hz}$ range have to be included as well as microphonic perturbations and noise in the $\mathrm{kHz}$ range from digital components and switching power supplies. Commercially available rf measurement devices that can quantify the performance independently of the controller hardware cannot provide the needed relative amplitude accuracy of $10^{-5} \mathrm{rms}$. For that reason, the performance of the controller so far has been determined by analyzing its own digital signals only. A relative cross-check, using a second controller board to analyze the signals, will be performed soon.

The data acquisition software writes the data from the FPGA to hard disk for an off-line analysis with the full sampling rate of the ADCs over a period of one minute. An appropriate indicator for the performance is the residual error of the controllers. Since residual errors are represented by very small numbers, it is essential to use the full resolution of 18 bits for further analysis.

Figure 6 shows one second of a typical recorded error signal. The histogram of the signal has been computed from the full data set ( 60 seconds). It shows that the signal is nearly Gaussian distributed with a deviation of $2.4^{\circ} \mathrm{rms}$.

A good visualization of the different perturbation frequencies can be obtained by calculating the "integrated" spectrum [20],

$$
\left[\Delta \phi\left(f_{n}\right)\right]_{\mathrm{rms}}=\frac{\sqrt{\sum_{i=1}^{n}\left|\mathcal{F}[\Delta \phi(t)]_{i}\right|^{2}}}{\sqrt{2}},
$$

where $\left[\Delta \phi\left(f_{n}\right)\right]_{\mathrm{rms}}$ is the spectrum of the time series integrated up to the perturbation frequency $f_{n}$. As the signal is discrete the "integration" is carried out by cumulating the coefficients of the single-sided discrete Fourier transform $\mathcal{F}[\Delta \phi(t)]_{i}$ up to the perturbation frequency of index $n$. The integrated spectrum is given in Fig. 8(a). In contrast to the normal spectrum this representation makes it much easier to see how the total rms error is distributed over different frequencies, especially if the spectrum contains many very sharp peaks. 

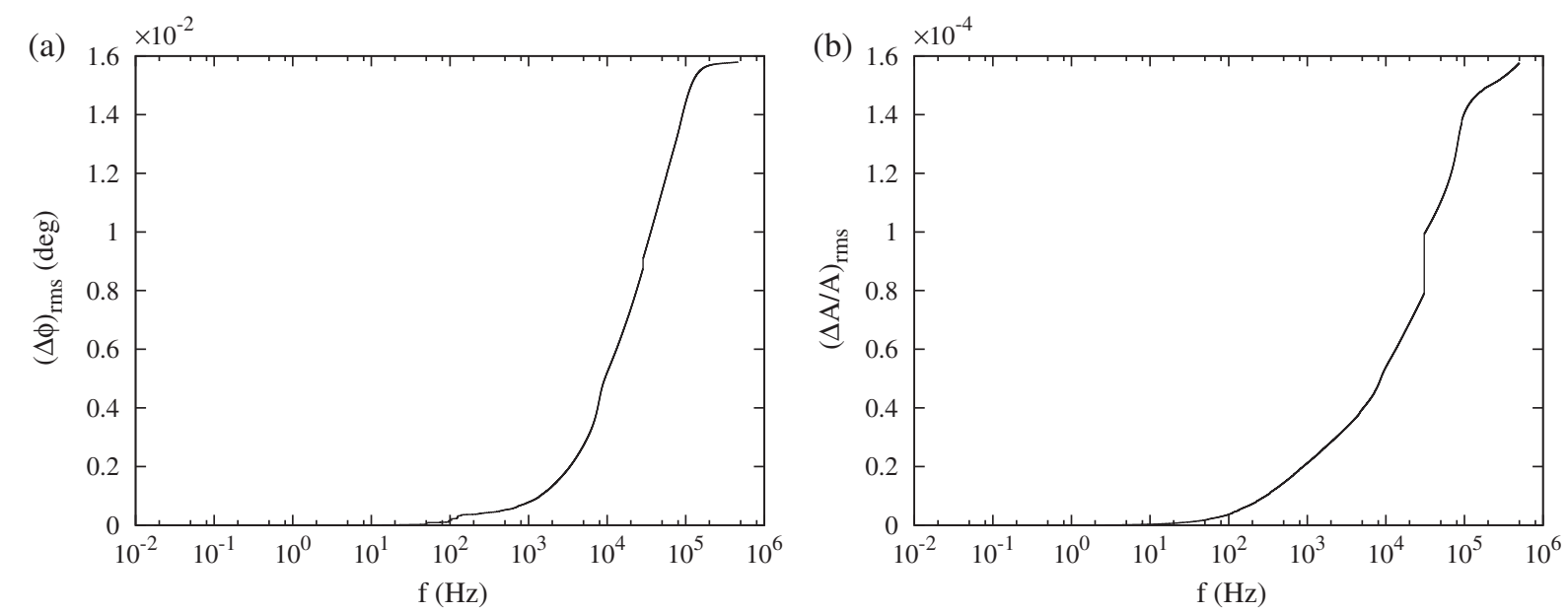

FIG. 7. Integrated spectra of (a) phase error and (b) amplitude error of the GDR. Amplitude and phase feedback are both active.
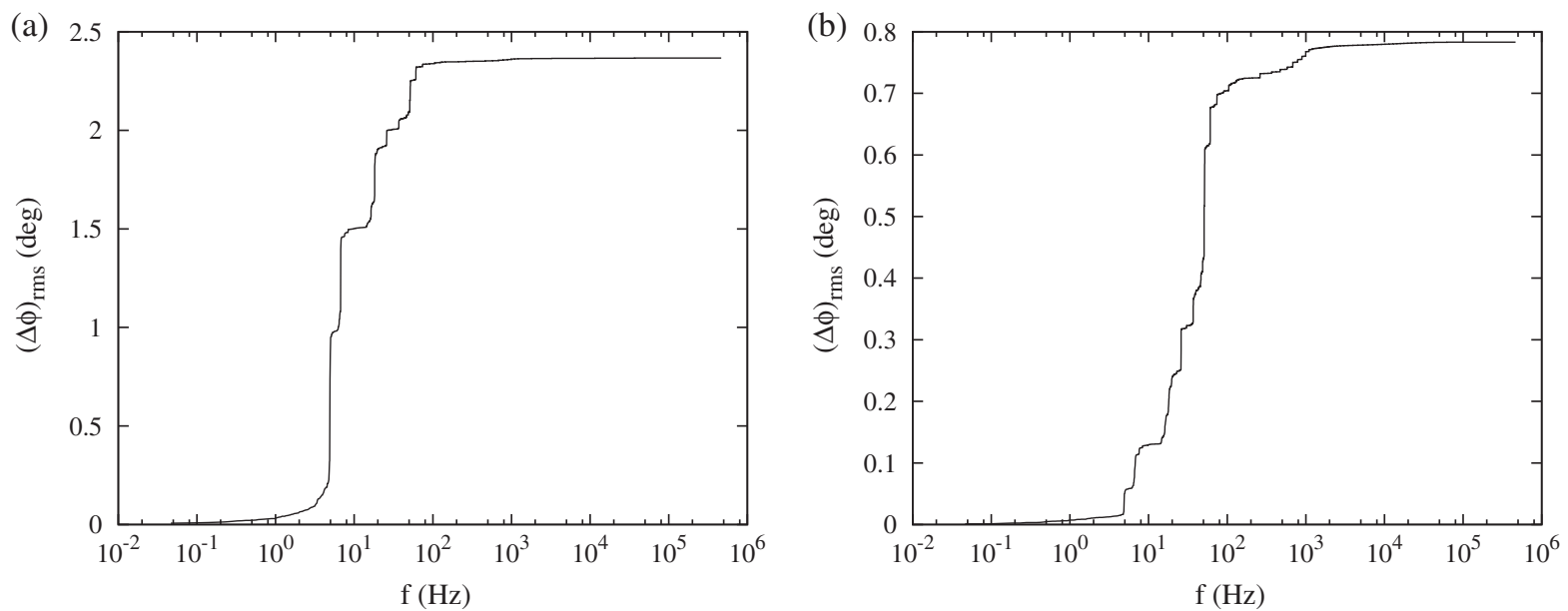

FIG. 8. Integrated magnitude spectra of phase error of the SEL with (a) amplitude and phase controllers deactivated and (b) both feedback loops closed.

\section{A. Generator-driven resonator}

The performance of the GDR algorithm used for the normal-conducting cavities has been measured with a copper resonator that is used for beam chopping. It has a loaded quality factor of approximately 5000 . The measurements have been performed with the same parameters that are used for beam operation. Because of the fact that the cavity is operated with up to $100 \mathrm{~W}$ rf power, it is cooled by water that is stabilized to $(20.0 \pm 0.1)^{\circ} \mathrm{C}$.

Integrated spectra of the residual errors are given in Fig. 7. Phase and amplitude feedback have both been activated while recording the data. The total phase error of $0.016^{\circ} \mathrm{rms}$ is very close to the phase noise of the controller hardware itself and is much better than our requirements. The increase of the integrated spectrum at frequencies above $10 \mathrm{kHz}$ is caused by noise. The plateau above $100 \mathrm{kHz}$ results from the antialiasing filter that damps higher frequencies.
The relative amplitude error of $1.6 \times 10^{-4} \mathrm{rms}$ is higher than the results achieved with superconducting cavities because of the much bigger bandwidth of the normal-conducting cavity that results in more transmitted noise. Although the amplitude error is larger than with superconducting cavities, it still meets the specification.

Results achieved with the $6 \mathrm{GHz}$ control loop are similar to the performance at $3 \mathrm{GHz}$. Table I gives an

TABLE I. Residual phase and amplitude errors accomplished with both phase and amplitude feedback loops closed.

\begin{tabular}{lccc}
\hline \hline Control algorithm & Operating frequency & $(\Delta \phi)_{\mathrm{rms}}$ & $(\Delta A / A)_{\mathrm{rms}}$ \\
\hline GDR & $3 \mathrm{GHz}$ & $0.016^{\circ}$ & $1.6 \times 10^{-4}$ \\
GDR & $6 \mathrm{GHz}$ & $0.012^{\circ}$ & $3.7 \times 10^{-4}$ \\
SEL & $3 \mathrm{GHz}$ & $0.78^{\circ}$ & $7.2 \times 10^{-5}$ \\
\hline \hline
\end{tabular}



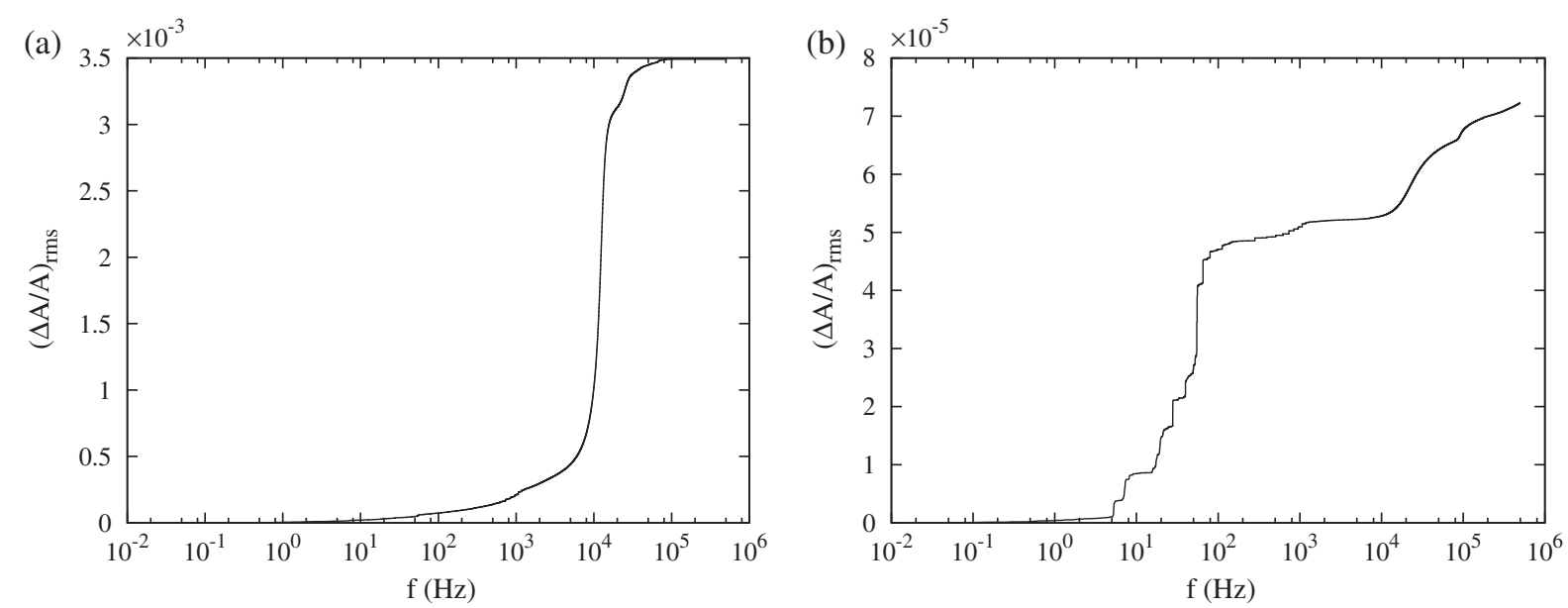

FIG. 9. Integrated magnitude spectra of relative amplitude error of the SEL with (a) phase controller activated but without amplitude feedback and (b) with both feedback loops closed. Parameters have been optimized for small residual errors with both controllers enabled.

overview of the residual errors obtained at both operating frequencies.

\section{B. Self-excited loop}

The performance of the SEL algorithm used for the superconducting cavities has been measured operating a 20-cell cavity in the cryomodule of the S-DALINAC at a typical field strength of 4 to $5 \mathrm{MV} / \mathrm{m}$. Figure 8 (a) shows the integrated phase error during SEL operation while all controllers are switched off. The total magnitude of the phase fluctuations is $2.4^{\circ} \mathrm{rms}$. By turning on the phase controller, these fluctuations can be reduced to a value below $0.3^{\circ} \mathrm{rms}$ [21]. If both phase and amplitude controllers are active, it becomes more difficult to reduce phase as well as amplitude fluctuations. A trade-off between phase and amplitude errors has to be made. In Fig. 8(b) the parameters of the controllers have been chosen to damp the phase fluctuations to a value of roughly $0.7^{\circ} \mathrm{rms}$ which is our target specification. Figure 9(b) shows the corresponding amplitude error that has been recorded at the same time. Compared to Fig. 9(a), the amplitude controller reduces the amplitude fluctuations significantly to $\Delta A / A=7.2 \times 10^{-5} \mathrm{rms}$ being also in accord with specifications.

The source of the noticeable high amplitude error in Fig. 9(a) at approximately $10 \mathrm{kHz}$ is still unknown. The perturbations only appear if the phase controller is active (with the same parameters that were optimized for small residual errors with both controllers enabled), while the amplitude controller is switched off.

\section{Detector operation}

During commissioning it turned out that the rf control system is sensitive enough to roughly detect the phase of the field in a switched off superconducting cavity induced by an electron beam of about $1 \mu \mathrm{A}$ (pick-up operation). This allows the operator to quickly determine the correct phase relationship between consecutive cavities when the distance between cavities has changed, e.g., due to maintenance of a cryomodule.

In addition, the rf control hardware has been used as a part of a detection system for longitudinal dispersion based on phase measurements on rf monitors [22]. Within this detection system the longitudinal dispersion is determined measuring small changes in rf phase of the passing beam precisely. This allows one to tune the accelerator to different nonisochronous working points to reduce the energy spread in future.

\section{SUMMARY}

The S-DALINAC's new digital rf control system is based on hardware developed in-house giving us full control over every detail of the implementation. The soft CPU that has been implemented in the FPGA allows fast and flexible modification of the control algorithm on one hand and powerful diagnostics on the other hand. The availability of extensive diagnostics has proven to be a considerable precondition for the smooth commissioning of a new $\mathrm{rf}$ control system. The highly automatized deployment of all parts of the software and configuration yielded a fast integration of further improvements into the production system.

The rf control system meets its specifications in SEL mode as well as GDR mode. It can be used to detect the phase of the electron beam during commissioning and, in addition to this, the hardware has been used in a detection system for longitudinal dispersion. During the first year of operation the rf control system has proven to be very reliable. Development of a pulsed variant of the presented system is under way for a $325 \mathrm{MHz}$ cavity at the p-LINAC test stand at GSI/FAIR [23].

The presented results demonstrate that the base-band approach represents a viable option for rf control systems even in the case of high demands on accuracy. 


\section{ACKNOWLEDGMENTS}

The development of the new rf control system for the S-DALINAC has been supported by DFG through CRC 634.

[1] A. Richter, in Proceedings of European Particle Accelerator Conference 1996, Barcelona, Spain (Institute of Physics, London, 1996), pp. 110-114.

[2] C. Eckardt, T. Bahlo, P. Bangert, R. Barday, U. Bonnes, M. Brunken, C. Burandt, R. Eichhorn, J. Enders, M. Espig, C. Ingenhaag, J. Lindemann, M. Platz, Y. Poltoratska, M. Roth, F. Schneider, H. Schüßler, M. Wagner, A. Weber, and B. Zwicker, in Proceedings of the 2011 Particle Accelerator Conference, New York, USA (IEEE, New York, 2011) pp. 853-855.

[3] D. Savran, K. Lindenberg, J. Glorius, B. Löher, S. Müller, N. Pietralla, L. Schnorrenberger, V. Simon, K. Sonnabend, C. Wälzlein, M. Elvers, J. Endres, J. Hasper, and A. Zilges, Nucl. Instrum. Methods Phys. Res., Sect. A 613, 232 (2010).

[4] N. Ryezayeva, H. Arenhövel, O. Burda, A. Byelikov, M. Chernykh, J. Enders, H. W. Grießhammer, Y. Kalmykov, P. von Neumann-Cosel, B. Özel, I. Poltoratska, I. Pysmenetska, C. Rangacharyulu, S. Rathi, A. Richter, G. Schrieder, A. Shevchenko, and O. Yevetska, Phys. Rev. Lett. 100, 172501 (2008).

[5] O. Burda, N. Botha, J. Carter, R. W. Fearick, S. V. Förtsch, C. Fransen, H. Fujita, J. D. Holt, M. Kuhar, A. Lenhardt, P. von Neumann-Cosel, R. Neveling, N. Pietralla, V. Y. Ponomarev, A. Richter, O. Scholten, E. Sideras-Haddad, F. D. Smit, and J. Wambach, Phys. Rev. Lett. 99, 092503 (2007).

[6] Xinping Huang and M. Caron, in International Symposium on Circuits and Systems, ISCAS, 2002 (IEEE, New York, 2002), Vol. 4, pp. 811-814.

[7] A. Araz, U. Bonnes, R. Eichhorn, F. Hug, M. Konrad, M. Platz, A. Richter, and R. Stassen, Phys. Rev. ST Accel. Beams 13, 082801 (2010).

[8] J. Volder, IRE Transactions on Electronic Computers (IRE, New York, 1959), pp. 330-334.
[9] J. Delayen, Ph.D. thesis, California Institute of Technology, 1978.

[10] J. Delayen, T. Allison, C. Hovater, J. Musson, and T. Plawski, in Proceedings of SRF, Peking, China, 2007, pp. 230-234.

[11] Xilinx Inc., "LogiCORE IP CORDIC v4.0 — product specification" [http://www.xilinx.com/support/documentation/ ip_documentation/cordic_ds249.pdf].

[12] "The Ethernut project" [http://www.ethernut.de].

[13] "The SocketCAN project" [http://developer.berlios.de/ projects/socketcan/].

[14] X. Chen and K. Kasemir, in Proceedings of the 2011 Particle Accelerator Conference, NY, USA (Ref. [2]), pp. 1404-1406.

[15] J. Hatje, M. Clausen, Ch. Gerke, M. Moeller, and H. Rickens, in Proceedings of the International Conference on Accelerator and Large Experimental Physics Control Systems, Knoxville, 2007, pp. 37-39.

[16] "Osqoop. The open oscilloscope" [http://gitorious.org/ osqoop].

[17] SigBlips, "Baudline. A time-frequency browser" [http:// www.baudline.com].

[18] "FAI. The Debian Fully Automatic Installation project" [http://fai-project.org].

[19] Xilinx Inc., "Spartan-6 FPGA configuration-user guide" [http://www.xilinx.com/support/documentation/user_guides/ ug380.pdf].

[20] A. Neumann, W. Anders, O. Kugeler, and J. Knobloch, Phys. Rev. ST Accel. Beams 13, 082001 (2010).

[21] M. Konrad, U. Bonnes, C. Burandt, R. Eichhorn, and N. Pietralla, in Proceedings of Linear Accelerator Conference, Tsukuba, Japan, 2010, pp. 268-270.

[22] F. Hug, C. Burandt, R. Eichhorn, and M. Konrad, in Proceedings of DITANET, Seville, Spain, 2011 (unpublished).

[23] R. Eichhorn, A. Araz, U. Bonnes, F. Hug, M. Konrad, P. Nonn, R. Stassen, G. Schreiber, and W. Vinzenz, in Proceedings of the International Particle Accelerator Conference, Kyoto, Japan (ICR, Kyoto, 2010), pp. 1413-1415. 